\title{
Tendencia y causas de mortalidad perinatal en el Hospital Hipólito Unanue de Tacna
}

\section{Trend and causes of perinatal mortality at Hipolito Unanue Hospital in Tacna}

\author{
Simón Eduardo Jimenez Estaña ${ }^{\text {1a }}$ \\ Manuel Ticona Rendón ${ }^{2 \mathrm{~b}}$ \\ ${ }^{1}$ Investigador independiente. Tacna, Perú. \\ ${ }^{2}$ Escuela de Medicina Humana de la Universidad Nacional Jorge Basadre. Tacna, Perú. \\ ${ }^{a}$ Médico Cirujano. \\ ${ }^{\mathrm{b}}$ Docente, Doctor en Salud Pública.
}

\section{RESUMEN}

La mortalidad perinatal es un indicador del control prenatal y calidad de atención de los servicios de salud para el feto y el recién nacido. Objetivo: Determinar la tendencia y las causas de la mortalidad perinatal. Material y métodos: Estudio descriptivo, retrospectivo y transversal, se incluyó a todos los nacimientos atendidos en el Hospital Hipólito Unanue de Tacna durante el período 2000-2018, con edad gestacional de 22 semanas a más o 500 gramos a más, cuya defunción fuese hasta el vigésimo octavo día de vida en neonatos. Se calculó tasa de mortalidad por mil nacimientos. Los datos se obtuvieron de la Unidad de Epidemiología. Para el procesamiento y análisis estadístico se utilizó el software Microsoft Excel 2007. Resultados: La tasa de mortalidad perinatal I calculada fue 14,9 por 1000 nacimientos y la tasa de mortalidad perinatal II fue 18,9 por 1000 nacimientos. Existe una tendencia a la disminución del riesgo de muerte perinatal. La muerte neonatal precoz contribuye a la muerte perinatal I más que la muerte fetal tardía. La muerte neonatal precoz se produce en mayor porcentaje en las primeras 24 horas de vida. Las causas de muerte neonatal fueron: síndrome de dificultad respiratoria, infecciones, malformaciones congénitas y asfixia perinatal. Conclusión: La tendencia de mortalidad perinatal es descendente, siendo las causas predominantes dificultad respiratoria, infecciones y malformaciones.

Palabras Clave: Mortalidad Fetal, mortalidad neonatal, mortalidad perinatal.

\begin{abstract}
Perinatal mortality is an indicator of prenatal control and quality of care of health services for the fetus and the newborn. Objective: To determine the tendency and causes of perinatal mortality. Material and methods: Descriptive, retrospective and cross-sectional study included all births attended at the Hipólito Unanue de Tacna Hospital during the 2000-2018 period, with gestational age of 22 weeks to more or 500 grams or more, whose death was up to the twenty-eighth day of life in neonates. Mortality rate per thousand births was calculated. Data were obtained from the Epidemiology Unit. Microsoft Excel 2007 software was used for the statistical processing and analysis. Results: The perinatal mortality rate I was 14.9 per 1000 births and the perinatal mortality rate II was 18.9 per 1000 births. There is a tendency to decrease the risk of perinatal death. Early neonatal death contributes to perinatal death I more than late fetal death. Early neonatal death occurs in greater percentage in the first 24 hours of life. The causes of neonatal death were: respiratory distress syndrome, infections, congenital malformations and perinatal asphyxiation. Conclusion: The tendency of perinatal mortality is descending, the predominant causes being respiratory distress, infections and malformations
\end{abstract}

Keywords: Fetal mortality, neonatal mortality, perinatal mortality. 


\section{Introducción}

La oportunidad de supervivencia del recién nacido está influenciada por una interacción de factores genéticos y ambientales. Durante este periodo el producto de la concepción está sometido a una serie de riesgos que dependen fundamentalmente del ambiente materno en el cual crece y se desarrolla y del ambiente exterior que puede ser tan favorable o no como el ambiente materno (1-3).

El conocimiento de la mortalidad perinatal es un hecho de gran importancia porque permite medir el estado de salud del producto de la concepción. La mortalidad perinatal es un indicador que evidencia el nivel de desarrollo que tiene la atención prenatal y del recién nacido en una determinada área geográfica o en un servicio. Por lo tanto, proporciona una imagen del nivel de desarrollo y calidad de salud de los pueblos $(3,4)$.

Desde el punto de vista de estudio de la ocurrencia de enfermedad, expresar la mortalidad en términos cuantitativos puede señalar diferencias en el riesgo de morir de una enfermedad. Las tasas de mortalidad sirven para medir la gravedad de la enfermedad y nos pueden ayudar a determinar si las medidas terapéuticas y preventivas de una enfermedad se han vuelto efectivas con el paso del tiempo. Por este motivo, si queremos abordar el riesgo de morir, debemos observar referidas tasas $(1,2)$.

La mayoría de las muertes suceden por causas prevenibles y tratables, por lo que los esfuerzos globales deben enfocarse no sólo en métodos terapéuticos más eficaces y accesibles en recién nacidos prematuros críticamente enfermos, sino también en una eficiente vigilancia del embarazo, trabajo de parto y parto. Existen diferencias notables en la mortalidad entre naciones con altos recursos y las de recursos limitados, por lo que la salud neonatal debe abordarse de manera más eficaz para continuar el rápido descenso de la mortalidad infantil global (5).

En el presente estudio no se produjeron cambios en las codificaciones ni en las definiciones de enfermedad, además las muertes se codificaron siguiendo la clasificación internacional de enfermedades (ICD, de International Classification of Diseases). En cuanto a la calidad de los datos, fueron proporcionados por la Unidad de Epidemiología, área de estadística del Hospital Hipólito Unanue de Tacna, por lo que se podrían considerar como fiables, pero es el médico de turno el encargado de colocar la causa principal de muerte. Es importante además denotar que en la institución de estudio no existe la variable causa de muerte fetal. Un factor importante del cual no se puede estudiar por carencia de datos. Por lo tanto, existe un sesgo evidente de tomar como referencia las estadísticas hospitalarias, las cuales se plantean gracias a este estudio y se espera que se superen.

El objetivo de este estudio fue determinar la tendencia y las causas más importantes para mortalidad perinatal en el Hospital Hipólito Unanue de Tacna 2000-2018.

\section{Material y métodos}

Estudio descriptivo, retrospectivo y transversal. Se incluyó a todos los recién nacidos vivos cuyo nacimiento se realizó en el Hospital Hipólito Unanue de Tacna, con edad gestacional de 22 semanas a más según último periodo menstrual de la madre y/o ecografía precoz o peso de 500 gramos a más y cuya defunción sea hasta el vigésimo octavo día de vida en neonatos, durante el período 2000-2018; se registró un total de 64350 nacimientos, 63740 nacidos vivos, 610 muertes fetales y 606 muertes neonatales.

Los datos se obtuvieron de la Unidad de Epidemiología, Área de Estadística que recoge los datos de los servicios de Neonatología y Gineco-Obstetricia, previa autorización. Para el procesamiento y análisis de datos, se utilizó el software Microsoft Office Excel 2007. Además, se utilizó estadísticas descriptivas como frecuencias absolutas y relativas y tasas por mil.

\section{Resultados}

Según el registro de la Unidad de Epidemiología, Área de Estadística del Hospital Hipólito Unanue de Tacna desde el 01 de enero del 2000 hasta el 31 de diciembre de 2018. Registra un total de 64 350 nacimientos, 63740 nacidos vivos, 610 muertes fetales y 606 muertes neonatales.

En la Tabla 1 se observa que durante el periodo de estudio de 2000-2018 un total de 64350 nacimientos, de los cuales 63740 son nacidos vivos y 610 son muertes fetales; además de 606 son muertes neonatales. Así, se puede apreciar que la cantidad de mortalidad fetal y neonatal es semejante. Se observa claramente que el número absoluto de muerte perinatal está disminuyendo con el paso de los años. 
Tabla 1. Distribución de muerte fetal y tasa de mortalidad fetal en el Hospital Hipólito Unanue de Tacna 2000-2018

\begin{tabular}{|c|c|c|c|c|c|c|}
\hline & \multicolumn{2}{|c|}{$\begin{array}{l}\text { Muerte fetal } \\
\text { intermedia }\end{array}$} & \multicolumn{2}{|c|}{$\begin{array}{c}\text { Muerte fetal } \\
\text { tardía }\end{array}$} & \multirow{2}{*}{$\begin{array}{c}\text { Muerte } \\
\text { fetal total } \\
\mathrm{n}=610\end{array}$} & \multirow[t]{2}{*}{ Tasa de mortalidad fetal } \\
\hline & $\mathrm{n}=164$ & tasa & $\mathrm{n}=446$ & tasa & & \\
\hline 2000 & 8 & 3,2 & 32 & 13,0 & 40 & 16,2 \\
\hline 2001 & 4 & 1,5 & 27 & 9,9 & 31 & 11,4 \\
\hline 2002 & 7 & 2,2 & 30 & 9,4 & 37 & 11,5 \\
\hline 2003 & 12 & 3,7 & 33 & 10,2 & 45 & 13,9 \\
\hline 2004 & 12 & 3,4 & 32 & 9,2 & 44 & 12,6 \\
\hline 2005 & 13 & 3,5 & 28 & 7,6 & 41 & 11,1 \\
\hline 2006 & 8 & 2,3 & 24 & 6,8 & 32 & 9,0 \\
\hline 2007 & 12 & 3,3 & 24 & 6,6 & 36 & 9,9 \\
\hline 2008 & 9 & 2,5 & 28 & 7,9 & 37 & 10,4 \\
\hline 2009 & 7 & 1,9 & 22 & 6,0 & 29 & 7,9 \\
\hline 2010 & 11 & 3,1 & 21 & 5,9 & 32 & 9,0 \\
\hline 2011 & 8 & 2,4 & 16 & 4,7 & 24 & 7,1 \\
\hline 2012 & 9 & 2,6 & 23 & 6,5 & 32 & 9,1 \\
\hline 2013 & 7 & 2,0 & 21 & 5,9 & 28 & 7,9 \\
\hline 2014 & 11 & 3,3 & 19 & 5,7 & 30 & 9,1 \\
\hline 2015 & 7 & 2,0 & 15 & 4,3 & 22 & 6,3 \\
\hline 2016 & 4 & 1,2 & 16 & 4,7 & 20 & 5,9 \\
\hline 2017 & 10 & 2,8 & 18 & 5,1 & 28 & 8,0 \\
\hline 2018 & 5 & 1,5 & 17 & 5,1 & 22 & 6,5 \\
\hline Total & 164 & 2,5 & 446 & 6,9 & 610 & 9,5 \\
\hline
\end{tabular}

Se observa una disminución en el riesgo de morir del grupo de muerte fetal intermedia y muerte fetal tardía. Además, se observa una disminución de la tasa de mortalidad fetal total de 16,2 en el 2000 a 5,9 en 2016; con una disminución de 10,3. Se observa que durante el período de estudio hubo menor cantidad de muerte fetal intermedia que de muerte fetal tardía.

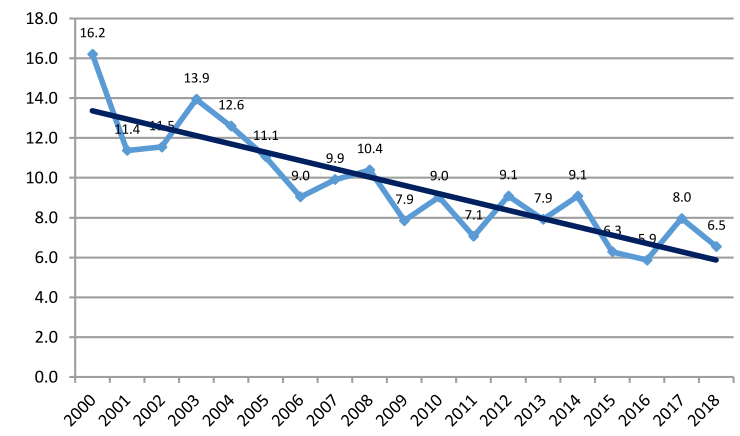

Figura 1. Tasa de mortalidad fetal en el Hospital Hipólito Unanue de Tacna 2000-2018 
Tabla 2. Distribución de muerte neonatal y tasa de mortalidad neonatal en el Hospital Hipólito Unanue de Tacna 2000 - 2018

\begin{tabular}{ccccccc}
\multicolumn{2}{c}{} & Muerte neonatal precoz & Muerte neonatal tardía & $\begin{array}{c}\text { Muerte } \\
\text { Neonatal }\end{array}$ & $\begin{array}{c}\text { Tasa de mortalidad } \\
\text { neonatal }\end{array}$ \\
2000 & $\mathrm{n}=514$ & TASA & $\mathrm{n}=92$ & TASA & $\mathrm{n}=606$ & 7,8 \\
2001 & 18 & 7,4 & 1 & 0,4 & 19 & 13,4 \\
2002 & 35 & 13,0 & 1 & 0,4 & 36 & 10,7 \\
2003 & 30 & 9,5 & 4 & 1,3 & 34 & 11,3 \\
2004 & 32 & 10,1 & 4 & 1,3 & 36 & 16,8 \\
2005 & 54 & 15,6 & 4 & 1,2 & 58 & 8,5 \\
2006 & 26 & 7,1 & 5 & 1,4 & 31 & 11,7 \\
2007 & 35 & 10,0 & 6 & 1,7 & 41 & 8,3 \\
2008 & 28 & 7,8 & 2 & 0,6 & 30 & 9,4 \\
2009 & 28 & 8,0 & 5 & 1,4 & 33 & 10,6 \\
2010 & 35 & 9,5 & 4 & 1,1 & 39 & 9,1 \\
2011 & 28 & 8,0 & 4 & 1,1 & 32 & 8,3 \\
2012 & 26 & 7,7 & 2 & 0,6 & 28 & 4,6 \\
2013 & 10 & 2,9 & 6 & 1,7 & 16 & 10,5 \\
2014 & 25 & 7,1 & 12 & 3,4 & 37 & 6,1 \\
2015 & 15 & 4,6 & 5 & 1,5 & 20 & 12,7 \\
2016 & 35 & 10,1 & 9 & 2,6 & 44 & 8,3 \\
2017 & 21 & 6,2 & 7 & 2,1 & 28 & 6,9 \\
2018 & 18 & 5,2 & 6 & 1,7 & 24 & 6,0 \\
TOTAL & 15 & 4,5 & 5 & 1,5 & 20 & 9,5
\end{tabular}

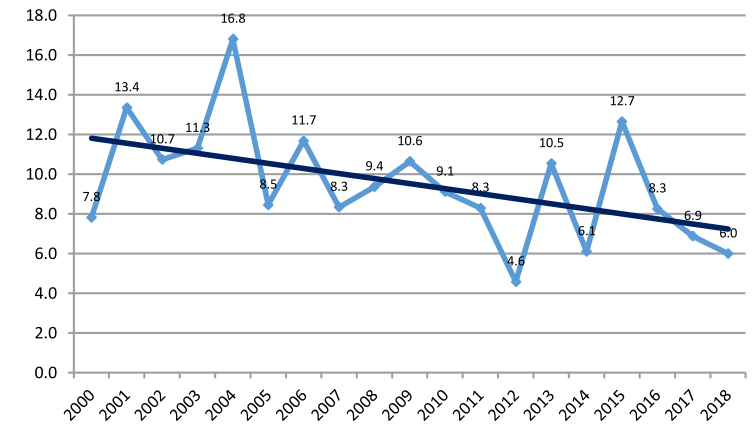

Figura 2. Tasa de mortalidad neonatal en el Hospital Hipólito Unanue de Tacna 2000-2018

En la tabla 3, se observa una disminución en la tendencia de la tasa de mortalidad perinatal I y II. De similar manera de un valor máximo de tasa de mortalidad perinatal II de 29,2 en 2004 a un valor de 12,5 en 2018.

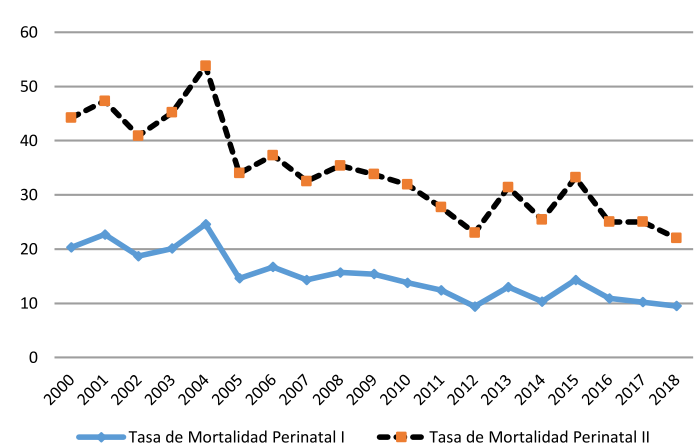

Figura 3. Tasa de mortalidad perinatal en el Hospital Hipólito Unanue de Tacna 2000-2018 
Tabla 3. Distribución de muerte perinatal y tasa de mortalidad perinatal en el Hospital Hipólito Unanue de Tacna 2000-018

\begin{tabular}{|c|c|c|c|c|}
\hline & $\begin{array}{l}\text { Muerte } \\
\text { perinatal I }\end{array}$ & $\begin{array}{c}\text { T. Mortalidad } \\
\text { perinatal I }\end{array}$ & $\begin{array}{c}\text { Muerte } \\
\text { Perinatal II }\end{array}$ & $\begin{array}{c}\text { T. Mortalidad } \\
\text { perinatal II }\end{array}$ \\
\hline & $\mathrm{n}=960$ & & $\mathrm{n}=1216$ & \\
\hline 2000 & 50 & 20,3 & 59 & 23,9 \\
\hline 2001 & 62 & 22,7 & 67 & 24,6 \\
\hline 2002 & 60 & 18,7 & 71 & 22,2 \\
\hline 2003 & 65 & 20,1 & 81 & 25,1 \\
\hline 2004 & 86 & 24,6 & 102 & 29,2 \\
\hline 2005 & 54 & 14,6 & 72 & 19,4 \\
\hline 2006 & 59 & 16,7 & 73 & 20,6 \\
\hline 2007 & 52 & 14,3 & 66 & 18,2 \\
\hline 2008 & 56 & 15,7 & 70 & 19,7 \\
\hline 2009 & 57 & 15,4 & 68 & 18,4 \\
\hline 2010 & 49 & 13,8 & 64 & 18,1 \\
\hline 2011 & 42 & 12,4 & 52 & 15,3 \\
\hline 2012 & 33 & 9,4 & 48 & 13,6 \\
\hline 2013 & 46 & 13,0 & 65 & 18,4 \\
\hline 2014 & 34 & 10,3 & 50 & 15,1 \\
\hline 2015 & 50 & 14,3 & 66 & 18,9 \\
\hline 2016 & 37 & 10,9 & 48 & 14,1 \\
\hline 2017 & 36 & 10,2 & 52 & 14,8 \\
\hline 2018 & 32 & 9,5 & 42 & 12,5 \\
\hline TOTAL & 960 & 14,9 & 1216 & 18,9 \\
\hline
\end{tabular}

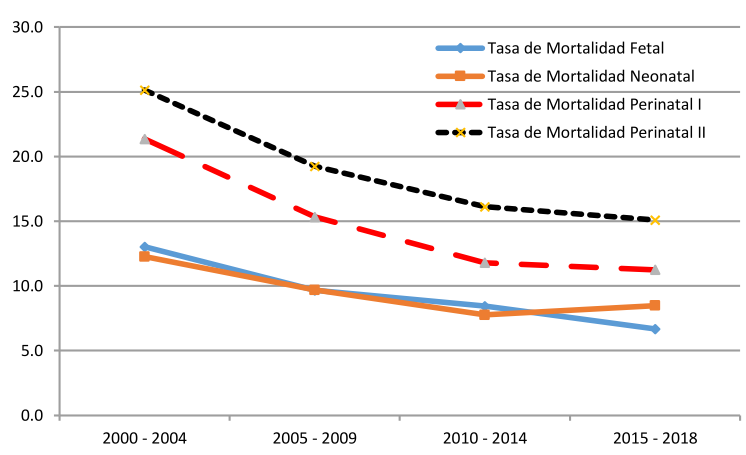

Figura 4. Tasa de mortalidad perinatal I y II en el Hospital Hipólito Unanue de Tacna 2000-2018.

En la Tabla 5 se observa que las 4 principales causas generales de muerte neonatal total son: síndrome de dificultad respiratoria $(40,6 \%)$, infecciones $(15,5$ $\%$, malformaciones congénitas o cromosómicas (14,7 $\%)$ y asfixia neonatal (10,7\%). Se observa además un número elevado en otras causas de muerte neonatal; donde predomina principalmente causa no específica de muerte neonatal.

Tabla 4. Causas generales de muerte neonatal en el Hospital Hipólito Unanue de Tacna 2000-2018

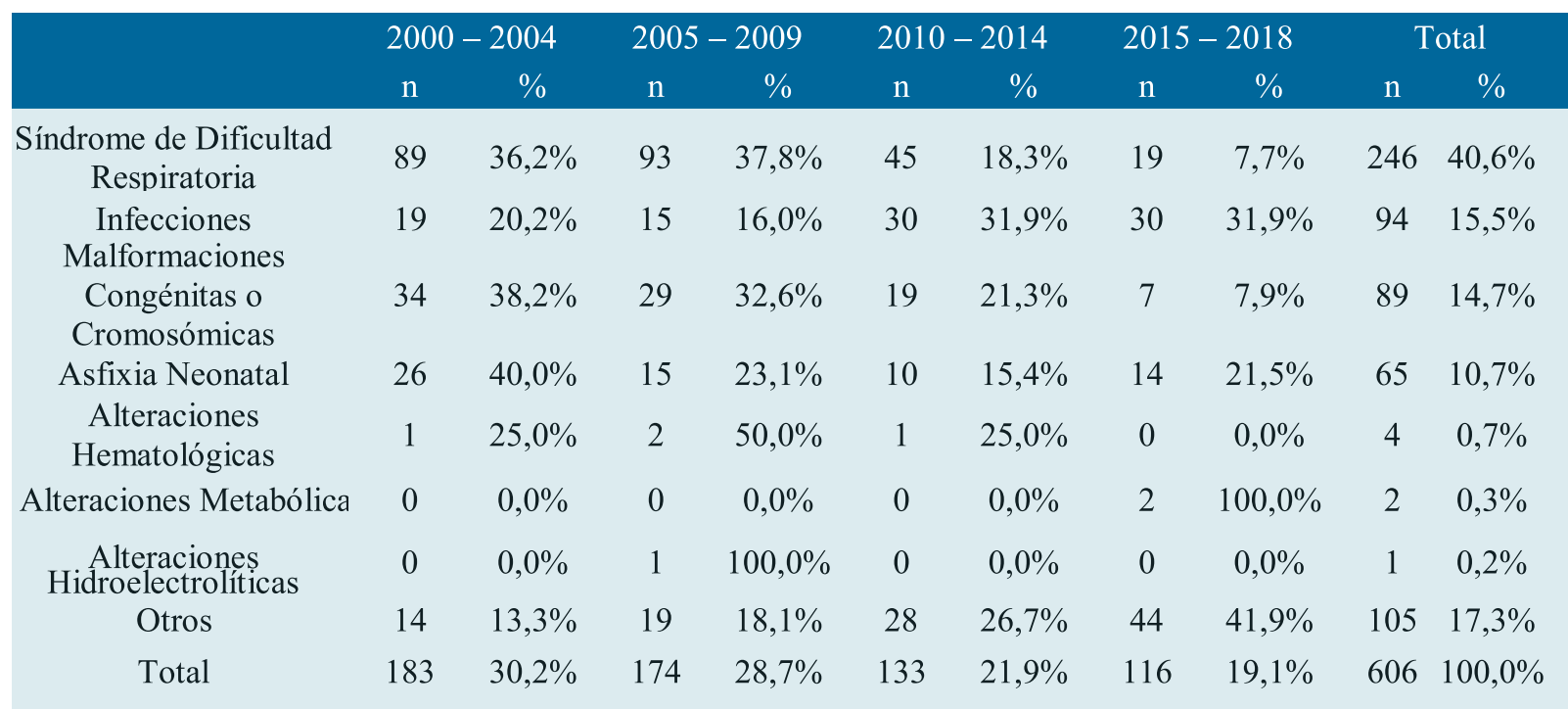




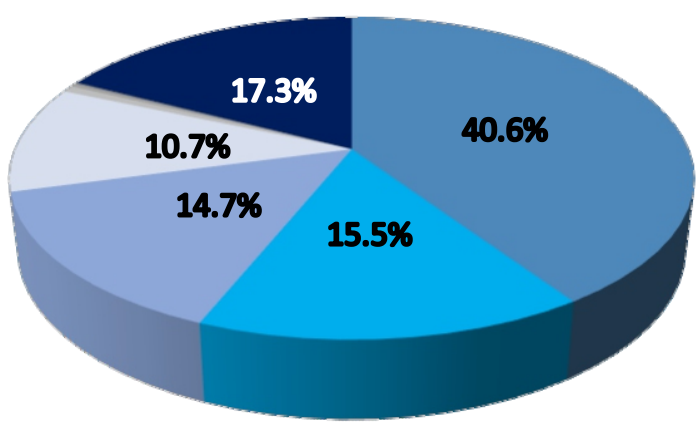

- Síndrome de Dificultad Respiratoria

- Infecciones

- Malformaciones Congénitas o

Cromosómicas

Asfixia Neonatal

Alteraciones Hematológicas

Alteraciones Metabólicas

- Alteraciones Hidroelectrolíticas

- Otros

Figura 5. Causas generales de mortalidad neonatal en el Hospital Hipólito Unanue de Tacna 2000-2018

Tabla 5. Tiempo de vida del neonato en el Hospital Hipólito Unanue de Tacna 2000-2018

\begin{tabular}{cccccccccccc} 
& \multicolumn{2}{c}{$2000-2004$} & \multicolumn{2}{c}{$2005-2009$} & $2010-2014$ & $2015-2018$ & \multicolumn{2}{c}{ Total } \\
& $\mathrm{n}=183$ & $\%$ & $\mathrm{n}=174$ & $\%$ & $\mathrm{n}=133$ & $\%$ & $\mathrm{n}=116$ & $\%$ & $\mathrm{n}=606$ & $\%$ \\
$<24 \mathrm{~h}$ & 88 & $48,1 \%$ & 78 & $44,8 \%$ & 48 & $36,1 \%$ & 36 & $31,0 \%$ & 250 & \\
$24-47 \mathrm{~h}$ & 26 & $14,2 \%$ & 26 & $14,9 \%$ & 15 & $11,3 \%$ & 14 & $12,1 \%$ & 81 & \\
$48-71 \mathrm{~h}$ & 15 & $8,2 \%$ & 13 & $7,5 \%$ & 14 & $10,5 \%$ & 4 & $3,4 \%$ & 46 & \\
$72 \mathrm{~h}-<7 \mathrm{~d}$ & 33 & $18,0 \%$ & 33 & $19,0 \%$ & 27 & $20,3 \%$ & 31 & $26,7 \%$ & 124 & \\
$7 \mathrm{~d}-28 \mathrm{~d}$ & 21 & $11,5 \%$ & 24 & $13,8 \%$ & 29 & $21,8 \%$ & 31 & $26,7 \%$ & 105 & \\
Total & 183 & $100,0 \%$ & 174 & $100,0 \%$ & 133 & $100,0 \%$ & 116 & $100,0 \%$ & 606 & $100,00 \%$
\end{tabular}

En la Tabla 6 se observa $41,25 \%$ de las muertes neonatales ocurren en las primeras 24 horas de vida, y ha disminuido en 17,1 puntos porcentuales desde el primer quinquenio al cuarto quinquenio.

\section{Discusión}

Con respecto a la muerte fetal, la literatura documenta que con los años se observa la disminución de la tasa de mortalidad fetal; la tendencia en disminución de las muertes fetales, así como disminución de la tasa de mortalidad fetal total de 16,2 a 5,9. Con un mayor porcentaje de disminución de la tasa de mortalidad fetal tardía en comparación a la tasa de mortalidad fetal intermedia. En Nueva Zelanda, Norris et al. (6) presenta una reducción en la tasa de muerte fetal de 5,6 a 5,5 por 1000 nacimientos totales en 2014. Sin embargo, las muertes fetales de 28 semanas de gestación se han reducido mucho más, con una tasa anual de 2,8 \%. En el Reino Unido se observó un descenso de 5,75 por 1000 nacimientos totales en 1993 a 4,64 por 1000 nacimientos totales en 2013 (6). Hernández et al. (7) también demuestra en su estudio una tasa de mortalidad fetal de 11,2 por 1000 nacimientos, los cuales han descendido en un $57 \%$ en el periodo de estudio. Además, según el Ministerio de Salud en su Boletín Epidemiológico (8) se muestra que la mortalidad intraparto tiene una tendencia decreciente, en el año 2011 fue de $16,8 \%$ y que 7 de cada 10 defunciones fetales ocurren en el tercer trimestre. Nuestros resultados son apoyados con los trabajos de Richard, P., quien informa que durante los últimos 20 años la tasa de mortalidad fetal mayor o igual a 28 semanas ha disminuido $(-18,5 \%)$. 
Con respecto a la muerte neonatal, la literatura también documenta que con los años se observa una disminución de la tasa de mortalidad neonatal; nuestros resultados demuestran una disminución de la tasa de mortalidad neonatal precoz de 15,6 en 2004 a 4,5 en 2018. Y un aumento en la tasa de mortalidad neonatal tardía de 1,3 en 2002 a 1,5 en 2018. Estos datos son semejantes al estudio de Ticona y Huanco (4), quienes informan que en los últimos 15 años la mortalidad neonatal ha disminuido, llegando al $55 \%$ de la mortalidad infantil; asimismo Gonzales et al. (5) en su estudio informa que la tasa de mortalidad neonatal en el 2011 fue de 8,7 por 1000 nacidos vivos, y en 2014 de 8,1 con lo cual hubo un descenso de 6,9\%. Según las publicaciones de UNICEF (9), se describe que en el mundo la tasa de mortalidad neonatal ha descendido en un $47 \%$; específicamente, desde 36 muertes por cada 1000 nacidos vivos en 1990 hasta 19 muertes por cada 1000 nacidos vivos en 2015. Además, que la mortalidad neonatal disminuyó en un $41 \%$ entre 2000 y 2017. Nuestros resultados también son apoyados con los trabajos de Cassidy (8), quien informa que durante los últimos 20 años la tasa de mortalidad neonatal ha disminuido (-85,4\%).

En relación a la muerte perinatal, la literatura denota disminución de la tasa de mortalidad perinatal I, la cual es utilizada por el Centro Nacional de Estadísticas de Salud y la Organización Mundial de la Salud para hacer comparaciones internacionales. En nuestro estudio, se observa una disminución en las tasas de mortalidad perinatal I de 24,6 en 2004 a 9,5 en 2018. Además, que de los componentes de la mortalidad perinatal, se aprecia un aumento en el número de casos de muerte neonatal precoz. Estos datos se correlacionan con el estudio de Hernández (7), quien informa que la tasa de muerte perinatal fue de 20,5 por cada 1000 nacimientos, con una reducción significativa de 48,4 \% de 1972 a 2006; es decir, 27,8 por cada 1000 nacimientos. Este hallazgo también se apoya con los trabajos de Cassidy (8) quien informa que durante los últimos 20 años la tasa de mortalidad perinatal ha disminuido a 7,5 por mil nacimientos. En el Boletín Informativo del Ministerio de Salud, se describe un incremento en la tendencia de mortalidad perinatal entre los años 2011 y 2013. Esta premisa también se puede observar en nuestro estudio, pero que al paso de los años la tendencia sigue disminuyendo, aunque observamos una mayor tasa en nuestro estudio. Además, en cuanto a la mortalidad perinatal II también se observa un menor número de casos de parte de las muertes fetales intermedias (164) que de la muerte neonatal tardía
(446). Asimismo, existe un crecimiento proporcional con el paso de los años en los casos de muerte neonatal total sobre el de muerte fetal total, pero con una proporción mayor aún de muerte fetal total. Según el estudio de Cassidy (8) este nos refiere que la infradeclaración de muertes fetales entre las semanas 22 y 27 parece ser severa (alrededor del $50 \%$ ), además al incluir las interrupciones del embarazo $\geq 22$ semanas, el número total aumenta de 2366 a 3080 casos y existe un incremento de la tasa de mortalidad perinatal de 5,7 por 1000 a 7,5 por 1000 nacimientos.

En cuanto a las causas fetales, estas no se pudieron analizar debido a que la Unidad de Epidemiología, Área de Estadística; no recaba esa variable: causa de muerte fetal.

En relación a las causas generales de muerte neonatal; la literatura refiere diversas causas de muerte; en nuestro estudio de investigación, se describe que en el periodo 2000 a 2018 , las principales causas de muerte neonatal fueron: síndrome de dificultad respiratoria $(40,6 \%)$, infecciones $(15,5 \%)$, malformaciones congénitas o cromosómicas $(14,7 \%$ ) y asfixia neonatal $(10,7 \%)$. Estos datos se correlacionan con los de Ticona y Huanco (4), quienes en su estudio refieren que, en el primer mes de vida, los recién nacidos fallecen principalmente por asfixia, distrés respiratorio, infección o malformaciones congénitas. Por otro lado, Gonzales et al. (5) difiere e informa que las causas básicas de mortalidad más frecuentes fueron prematurez y sus complicaciones, eventos de hipoxiaasfixia, sepsis bacteriana y malformaciones congénitas. Babaei et al. (10) muestran en su estudio que las causas más frecuentes de muerte fueron el síndrome de dificultad respiratoria $190(33,6 \%)$ y la sepsis 128 (22,6\%). Según el Ministerio de Salud del Perú, mediante su publicación del Boletín Epidemiológico del Perú, publicaron que las principales causas de muerte neonatal estuvieron relacionadas a asfixia y atención del parto; el $42 \%$ de las defunciones ocurrieron entre los días 1 a 7 de vida, y se relacionaron a asfixia e infecciones, esta proporción se redujo a $37 \%$ en 2011; y las muertes neonatales tardías están relacionadas principalmente con infecciones (11). Se aprecia que las principales causas de muerte neonatal en relación al estudio de Ticona y Huanco (4) se siguen manteniendo, pero con patrón porcentual distinto.

En cuanto a la muerte neonatal según el momento de muerte; en nuestro estudio, el 41,3\% del número de muertes neonatales se producen antes de las 24 horas de vida, Un $41,4 \%$ se produce en el primer a 
séptimo día de vida, Un 17,3\% ocurre en el periodo neonatal tardío. Se analiza que, del periodo neonatal, existe mayor mortalidad en el periodo neonatal precoz, del cual en el primer día se produce la mayor cantidad de muertes. Estos datos se correlacionan con el Ministerio de Salud del Perú en su Boletín Informativo informó que el $36 \%$ de las defunciones neonatales ocurrió en las primeras 24 horas de vida. El $42 \%$ ocurrió entre los días 1 y 7 de vida. El resto (22\%) en la mortalidad neonatal tardía (11).

\section{Conclusiones}

Se concluye que las causas más importantes de muerte perinatal II, en base al componente de muerte neonatal fueron: síndrome de dificultad respiratoria (membrana hialina), infecciones (sepsis bacteriana), malformaciones congénitas o cromosómicas (malformación del sistema nervioso y cardiovascular) y asfixia perinatal en el Hospital Hipólito Unanue de Tacna 2000-2018. La tasa de mortalidad perinatal I fue de 14.9 por 1000 nacimientos y la tasa de mortalidad perinatal II fue de 18,9 por cada 1000 nacimientos en el Hospital Hipólito Unanue de Tacna 2000-2018. En relación a la tasa de mortalidad perinatal II existe una tendencia a la disminución del riesgo de muerte perinatal II.

\section{Referencias bibliográfica}

1. Gordis L. Epidemiology. ELSEVIER Saunders. Fifth Edition. 2014.

2. Katz D, Elmore J, Wild D, Lucan S. Jekel's Epidemiology, Biostatistics, Preventive Medicine, and Public Health. ELSEVIER Saunders. Fourth Edition. 2014

3. Ministerio de Salud. Mortalidad Neonatal en el Perú y sus departamentos 2011 - 2012. Diciembre, 2013.

4. Ticona M, Huanco D. Mortalidad Perinatal. Rev Per Ginecol Obstet. 2004. 50: 61 - 71 .

5. Gonzales-Pérez D. et al. Tendencia y causas de mortalidad neonatal en el Instituto Mexicano del Seguro Social 2011-2014, a nivel nacional. Revista Mexicana de Pediatría. 2016; 83(4): $115-123$.

6. Norris T, et al. Causes and temporal changes in nationally collected stillbirth audit data in high-resource settings, Seminars in Fetal \& $\mathrm{N}$ e o n a t a 1 Medicine 2017. http://dx.doi.org/10.1016/j.siny.2017.02.003

7. Hernández-Herrera R, et al. Mortalidad fetal, neonatal y perinatal en un hospital de ginecoobstetricia. Revisión de 35 años. Rev
Med Inst Mex Seguro Soc 2009; 47 (2): 353 356.http://www.medigraphic.com/pdfs/imss/i m-2009/im094b.pdf

8. Cassidy R. Estadísticas y tasas de la mortalidad intrauterina, neonatal y perinatal en España. Revista Muerte y Duelo Perinatal. Umamanita 2018; Número 4.

9. UNICEF. Levels \& trends in child mortality, report 2018 . Disponible en: ht tps://data.unicef.org/w pcontent/uploads/2018/10/Child-MortalityReport-2018.pdf

10. Babaei H, Dehghan M, Mohammadi Pirkashani L. Study of Causes of Neonatal Mortality and its Related Factors in the Neonatal Intensive Care Unit of Imam Reza Hospital in Kermanshah, Iran during (20142016). Int J Pediatr 2018; 6(5) Serial No.53

11. Ministerio de Salud. Centro Nacional de Epidemiología, Prevención y Control de E n f e $\mathrm{r} m$ e d a d e s. B O L E T Í N EPIDEMIOLÓGICO DEL PERÚ - SE. 1. 2017 . D i s p o n i b l e e n : http://www.dge.gob.pe/portal/docs/vigilancia /boletines/2017/01.pdf

\section{Correspondencia}

md.simonjimenez@gmail.com
Fecha de recepción: 05 de setiembre de 2019

Fecha de aceptación: 05 de noviembre de 2019 